\title{
A capacitive RF power sensor based on MEMS technology
}

\author{
L J Fernández, R J Wiegerink, J Flokstra, J Sesé, H V Jansen \\ and $M$ Elwenspoek
}

$\mathrm{MESA}^{+}$Research Institute, University of Twente, PO Box 217, 7500 AE Enschede, The Netherlands

E-mail: 1.j.fernandez@utwente.nl

Received 13 December 2005, in final form 8 March 2006

Published 28 April 2006

Online at stacks.iop.org/JMM/16/1099

\begin{abstract}
Wideband $100 \mathrm{kHz}-4 \mathrm{GHz}$ power sensors are presented, which are based on sensing the electrostatic force between an RF signal line and a suspended membrane. The electrostatic force, which is proportional to the square of the rms signal voltage and thus to the signal power, results in a displacement of the suspended membrane. This displacement is detected capacitively, allowing the sensing of the signal power with extremely low dissipative losses; therefore the sensor can be placed in a transmission line with negligible disturbance of the signal. Devices have been designed and fabricated successfully by aluminum surface micromachining using photoresist as the sacrificial layer. Optimization of the design with SONNET has resulted in measured return and insertion losses $\left(S_{11}\right.$ and $\left.S_{21}\right)$ better than $-30 \mathrm{~dB}$ and $-0.15 \mathrm{~dB}$, respectively, up to $4 \mathrm{GHz}$, and a sensitivity of $90 \mathrm{aF} \mathrm{mW}^{-1}$.
\end{abstract}

\section{Introduction}

Modern communication systems require low weight, volume and power consumption and a high level of integration with electronics. In this field, a significant contribution from RF MEMS is expected [1]. In this paper, a power sensor for RF signals realized in MEMS technology is presented. The power sensor is based on a new sensing principle recently proposed for measuring RF signal power [2]. This principle is based on sensing the attractive electrostatic force between the signal line and a free standing electrode that is suspended at a small distance from the signal line. A power sensor based on this principle has very interesting properties. Most important is the possibility of operating the same sensor from low frequency ac through to microwaves as a true wideband device extending the frequency range of available instrumentation (voltmeters, power meters, signal sources). Furthermore, the sensor does not absorb the signal. Instead, a 'through' sensor is realized, with almost no loss of signal. Most existing technology for measuring the power of RF signals is based on terminating devices, i.e., the power is lost after the measurement. Examples are microcalorimeters, bolometers and thermistor mounts. These devices are sensitive to ambient temperature changes, and have limited dynamic range and long settling times. Diode sensors have the advantage of greater dynamic range, but they are sensitive to temperature variations and have problems with impedance matching. The sensor presented in this paper is suitable for sensing inline RF power in a way that the signal is not lost during RF operation: the through mode of sensing. Furthermore, the sensor is relatively insensitive to changes in ambient temperature, although a careful design is needed to prevent self-heating due to dissipation of the RF signal. The dynamic range is in principle comparable to diode sensors; however, in practice it will be limited by the accuracy at which the membrane displacements can be detected. For the realized devices a resolution in the order of $0.5 \mathrm{~mW}$ was obtained, which is far from the theoretical noise limit.

\section{Principle of operation}

The basic operation of the RF power sensor is based on the attractive force between the two electrodes of a voltage controlled capacitor. Figure 1 shows a schematic picture of a parallel plate capacitor with one fixed and one movable plate. When a voltage is applied to the plates, the electrostatic force will pull the movable plate towards the fixed plate. This effect is well known and exploited in many microsensors and 


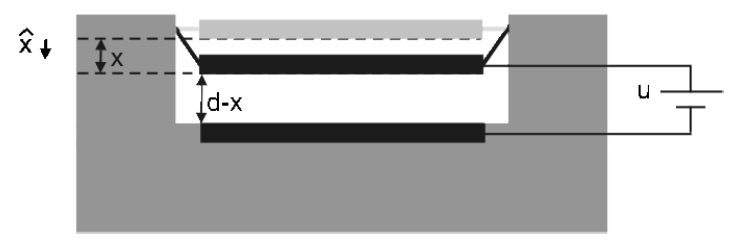

Figure 1. Schematic drawing of a parallel plate capacitor with one movable plate.

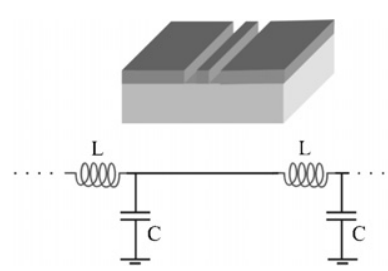

(a)

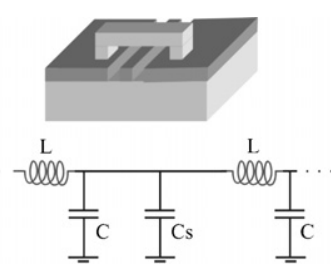

(b)
Figure 2. CPW and lumped element model $(a)$ and $\mathrm{CPW}$ with a movable capacitor model $(b)$.

microactuators. For a parallel plate configuration, the force can be expressed as

$$
\vec{F}_{\mathrm{el}}=\frac{1}{2} \frac{q^{2}}{\varepsilon A} \hat{x}=\frac{1}{2} \frac{\varepsilon A}{(d-x)^{2}} u^{2} \hat{x},
$$

as can be found in many textbooks, e.g. [3]. In this equation, $\varepsilon$ is the dielectric constant of the medium, $d$ is the initial gap between the plates, $x$ is the displacement of the movable plate, $A$ is the area of the plates, $q$ is the amount of charge on each plate and $u$ is the voltage between the plates. The voltage and charge are of course related by the capacitance of the structure:

$$
C=\frac{q}{u}=\frac{\varepsilon A}{d-x}
$$

When an ac voltage is applied between the capacitor electrodes, this will result in a time-dependent, but always attractive, electrostatic force. For frequencies well above the mechanical resonance frequency, only the average force is important, and the voltage $u$ in equation (1) is replaced by the rms voltage amplitude. Thus, by measuring the displacement of the movable capacitor plate or by measuring the force needed to keep the plate in its initial position, the (square of the) rms voltage amplitude of the ac signal can be obtained. This effect has been exploited to realize micro-mechanical rms-to-dc converters [4-7]. Seppä et al [2] proposed to exploit this principle for measuring the power level on transmission lines at very high frequencies, i.e., in the $\mathrm{GHz}$ range. In that case, one of the capacitor electrodes has to be replaced by an RF transmission line [8, 9]. The power of the RF signal is simply deduced from $P=V^{2} / Z$, where $P$ and $V$ are the power and rms voltage level of the signal, and $Z$ the impedance of the waveguide. Since the signal power and displacement of the movable membrane are both proportional to the square of the voltage level, a linear relation between power and displacement is found.

\section{Basic sensor structure}

Figure 2 shows a schematic drawing of a transmission line with and without sensor structure. For an ideal transmission

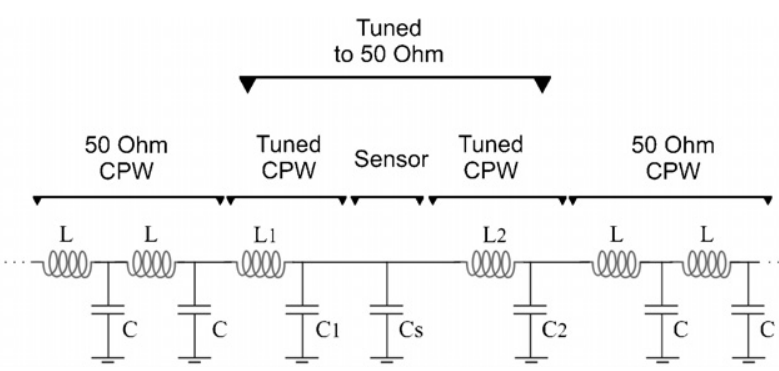

Figure 3. Schematic based on lumped elements theory showing the operation principle for the matching optimization of a capacitive power sensor.

line, the relation between the inductance and capacitance is fixed and, thus, the characteristic impedance is constant. In such a case, the RF energy transported is equally stored in the electric and the magnetic fields and no net forces exist between the conductors because the attraction due to the electric field is balanced by the repulsion due to the magnetic field [10]. A net force arises when a capacitive discontinuity is introduced in the transmission line, because a region is created where magnetic fields can be neglected in comparison with the electric fields. More important, when this capacitor has a movable plate (see figure 1) RF power can be sensed by detecting the deflection.

\subsection{Bandwidth}

As mentioned above, the sensor should be operated well above the mechanical resonance frequency. For the devices presented in this paper, this means that the lower frequency limit is around $100 \mathrm{kHz}$. The upper frequency limit is defined by the added capacitance. The insertion of a capacitor $C_{S}$ (see figure 2) in the transmission line changes its characteristic impedance and inevitably results in reflections and transmission losses. Because of this capacitance, the characteristic impedance of the transmission line seen at the position of the sensor, $Z_{L}$, becomes

$$
Z_{L}=Z_{C} \| Z_{0}=\frac{1}{j \omega C_{S}+1 / Z_{0}},
$$

where $Z_{0}$ is the initial characteristic impedance of the line $(50 \Omega)$ and $\omega$ is the angular frequency. Then, the voltage reflection coefficient can be calculated, giving

$$
\Gamma=\frac{Z_{L}-Z_{0}}{Z_{L}+Z_{0}}=\frac{1}{\frac{2}{j \omega C Z_{0}}+1} .
$$

Thus, the reflection losses increase with frequency and the maximum frequency for which the sensor can be used is defined by the capacitance value. Nevertheless, an increase in the sensor bandwidth can be realized by introducing compensating elements in the circuit around the sensor device [11]. Figure 3 illustrates how an extra series inductance $\left(L_{1}=L_{2}>L\right)$ and a reduced ground capacitance $\left(C_{1}=\right.$ $C_{2}<C$ ) implemented in the CPW surrounding the sensor can be used to retune the characteristic impedance of the total circuit to the desired $50 \Omega$. Of course, this only works as long as the dimensions of the circuit are much smaller than the wavelength of the signal. 


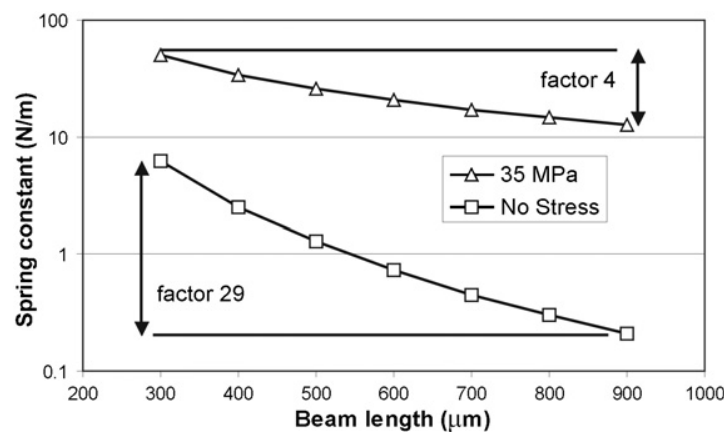

Figure 4. Comparison of spring constant estimation for clamped-clamped beams made of aluminum with different lengths, with and without stress in the structural material.

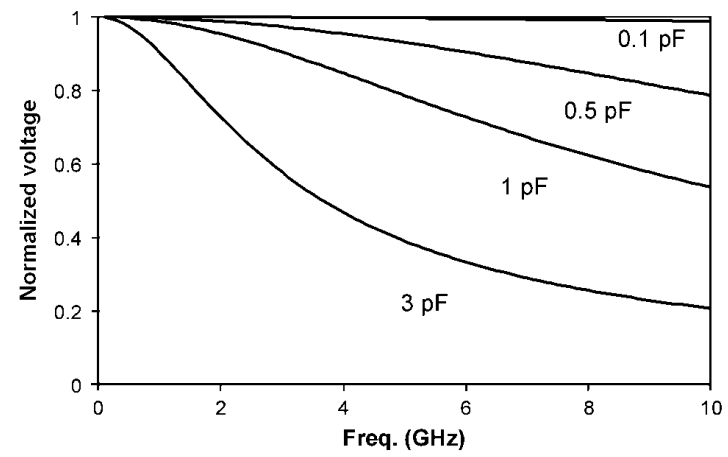

Figure 5. Normalized voltage across a shunt capacitance due to the presence of an RF signal as a function of frequency for different capacitance values.

\subsection{Sensitivity}

The sensitivity of an RF capacitive MEMS power sensor is determined by its capability to transfer the electrostatic force induced by the microwave signal into displacement of the movable plate which acts as a sensor. Therefore, a minimization of the spring constant is needed in order to optimize the power readout. For a clamped-clamped beam configuration, changing the thickness and length of the beam can give a very wide range of spring constant values. However, special care needs to be taken with respect to the stress in the material used. Figure 4 compares the calculation results of the spring constant [12] as a function of the beam length (from 300 to $900 \mu \mathrm{m}$ ) between a stress free beam and a beam with $35 \mathrm{MPa}$ residual tensile stress. As can be observed, the factor of 29 gained for a stress free beam is decreased down to 4 when a $35 \mathrm{MPa}$ tensile stress is present in the beam. Another important aspect is the decrease of the local voltage on the capacitor caused by the capacitance mismatch produced by the sensor. Due to the presence of a capacitive discontinuity, the voltage across the capacitor results in [13]

$$
V_{c}=\sqrt{\frac{4 Z_{0} P_{s}}{4+Z_{0}^{2} \omega^{2} C_{S}^{2}}},
$$

where $P_{S}$ is the power level of the RF signal. In this way, the sensitivity could be affected by the extra capacitance introduced by the sensor and the frequency of the signal to be detected. Nevertheless, this effect is only relevant for very high capacitance and/or frequency values as can be observed from figure 5.

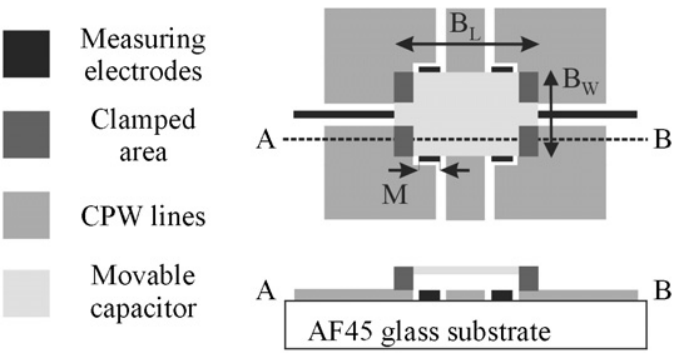

Figure 6. Schematic overview of a grounded RF power sensor design (top view and cross section).

3.3. Resolution and noise

The ultimate power resolution will be limited by the thermomechanical noise of the movable membrane. An estimate for the amount of noise can be obtained by considering the membrane as a second-order spring-mass system. In that case the mechanical noise force density acting on the mass is given by $[2,14]$

$$
\frac{\overline{F_{n}^{2}}}{\Delta f}=\frac{4 K k_{B} T}{\omega_{0} Q}=\frac{4 \sqrt{K M} k_{B} T}{Q},
$$

where $K$ is the mechanical spring constant, $k_{B} T$ the thermal energy, $\Delta f$ the readout frequency bandwidth, and $M, \omega_{0}$ and $Q$ are the mass, mechanical resonance frequency and quality factor. This noise force is shaped by the force-to-displacement transfer function of the system, just like the 'signal' force given by (1). Combining (1) and (6) gives the attainable power resolution. For the case of $1 \mathrm{~Hz}$ readout bandwidth, the minimum detectable power is

$$
S_{p}=\frac{u_{\mathrm{rms}, m}^{2}}{Z_{0}}=\frac{2 d}{C_{S} Z_{0}} \sqrt{\frac{4 \sqrt{K M} k_{B} T}{Q}} .
$$

Thus, to improve the power resolution we should decrease the air-gap $d$, spring constant $K$ and mass $M$ or increase the quality factor $Q$. The latter can be accomplished by operating the sensor in vacuum. Inserting the values for an aluminum beam $0.5 \mu \mathrm{m}$ thick, $500 \mu \mathrm{m}$ long, $50 \mu \mathrm{m}$ wide, with a $0.5 \mu \mathrm{m}$ gap, a quality factor of 100 and at $300 \mathrm{~K}$, a power resolution in the order of nanoWatts should be feasible.

\section{Sensor design and simulations}

We will distinguish between two different types of bridge designs regarding its connection to the transmission line. We will use a grounded bridge for the case where the membrane used as a movable capacitor is connected to the ground planes of the CPW, and a floating bridge for a design where the membrane is not electrically connected to the transmission line. We will treat the design of these two different types of capacitive sensors separately.

\subsection{Grounded bridge}

Figure 6 shows a schematic overview of a grounded bridge design. The bridge is connected at both ends to the ground planes of the CPW. Measuring electrodes, used as capacitive sensing elements, are placed under the bridge in between the 


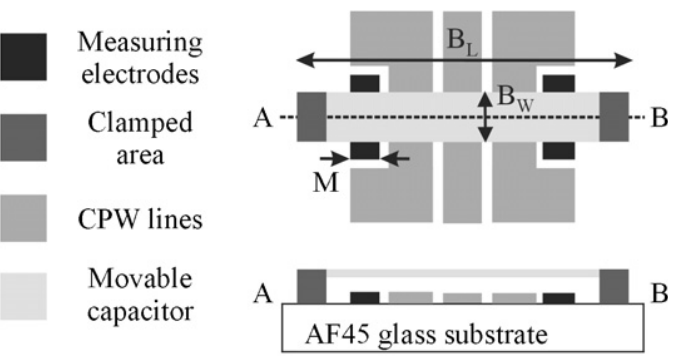

Figure 7. Schematic overview of a floating RF power sensor design: top view and cross section.

signal and ground planes. In order to get electrical access to the measuring electrodes the ground planes of the CPW were interrupted, but they remain electrically connected by the bridge. In this way, the measuring electrodes can be connected outside the sensing area. As a consequence of the design, the displacement detection is done by measuring the change in capacitance of a grounded capacitor, making it impossible to use accurate techniques like 'balanced-bridge' impedance measurements [15]. The beam was designed to be $300 \mu \mathrm{m}$ long $\left(B_{L}\right)$ with three different widths $\left(B_{W}\right), 200 \mu \mathrm{m}, 1.8 \mathrm{~mm}$ and $3.6 \mathrm{~mm}$. The measuring electrodes placed below are $50 \mu \mathrm{m}$ wide $(M)$ and as long as the width of the beam $\left(B_{W}\right)$.

\subsection{Floating bridge}

Figure 7 shows a schematic overview of a floating bridge design. The bridge is now much longer and extends beyond the ground planes of the CPW. The measuring electrodes are placed in between the ground planes and the clamp area of the bridge.

The expected sensitivity increases with respect to the grounded design because of the reduced spring constant (longer bridge), and the possibility of using a balanced-bridge impedance measurement for the capacitive readout, since an electrically floating capacitor is realized. Furthermore, the bandwidth of the sensor can be improved since lower capacitance values are required for the sensor. The beam was designed to be $900 \mu \mathrm{m}$ long $\left(B_{L}\right)$ with two different widths $\left(B_{W}\right), 110 \mu \mathrm{m}$ and $220 \mu \mathrm{m}$. The measuring electrodes placed below are $200 \mu \mathrm{m}$ wide $(M)$ and as long as the width of the beam $\left(B_{W}\right)$.

For the case of a floating bridge design, two different ways of impedance compensation techniques were investigated:

(1) Reducing the width of the signal line;

(2) Modifying the width of the ground planes.

In both cases, the gap distance of the CPW, $g_{\text {cpw }}(25 \mu \mathrm{m})$, is increased. As a result, lower capacitance and higher inductance are obtained in that region, compensating for the extra capacitance added by the sensor.

4.2.1. Reducing the width of the signal line. Figure 8 shows the detailed design and dimensions chosen for the realization of this impedance matching method. In this case, the central line width of the CPW, $w_{c p w}(100 \mu \mathrm{m})$, is reduced along a distance $d$, resulting in a new gap $g$, whereas the width of the ground planes, $g w_{\mathrm{cpw}}(300 \mu \mathrm{m})$, is maintained constant. In order to

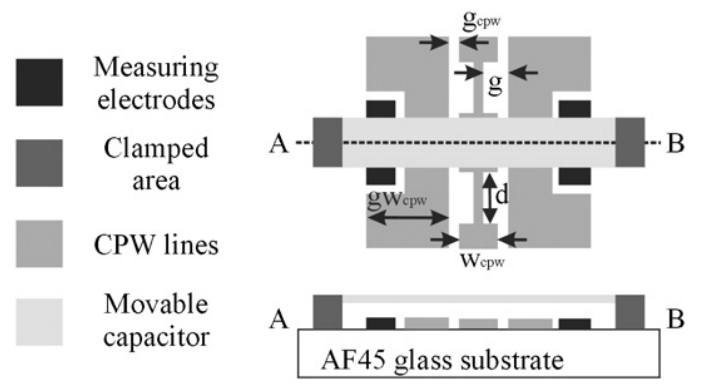

Figure 8. Schematic overview of a floating RF power sensor design: top view and cross section.

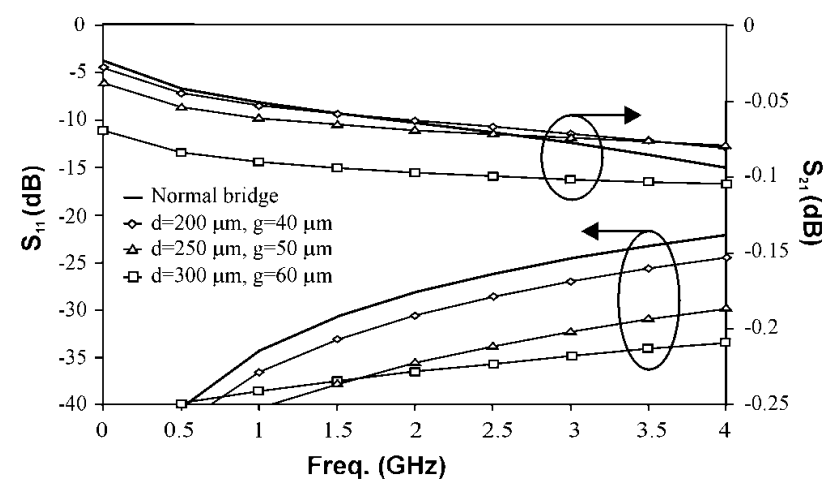

Figure 9. SONNET v10.52 simulation results for different designs of a compensated bridge sensor changing the width of the central line.

verify the operation principle of the method, simulations were performed using 'Sonnet v10.52' [16]. As can be observed from the simulation results of the return loss $\left(S_{11}\right)$ shown in figure 9, a better match is obtained when the width of the central line of the CPW is reduced.

The simulation results indicate that an improvement of $15 \mathrm{~dB}$ at $4 \mathrm{GHz}$ can be obtained. As a drawback, we can also observe that although reflections are lowered, the transmission loss increases when the signal line becomes too narrow $(w=30 \mu \mathrm{m})$. This is a consequence of the higher resistance introduced into the line, since the dimensions of the metal line which carries the RF current have been considerably reduced. Nevertheless, the magnitude of the insertion loss $\left(S_{21}\right)$ remains rather low, making it worthwhile to use this technique as a compensation method.

4.2.2. Modifying the width of the ground planes. Figure 10 shows the detailed design and dimensions chosen for the realization of this impedance matching method. In this case, the width of the ground planes, $g w_{\mathrm{cpw}}$, is reduced from its initial value $(300 \mu \mathrm{m})$ along a distance $d$, giving a new gap distance, $g$, whereas the width of the central line, $w_{\text {cpw }}$ $(100 \mu \mathrm{m})$, is maintained constant. The simulation results show an improvement of $15 \mathrm{~dB}$ at $4 \mathrm{GHz}$ in the return loss (see figure 11). Moreover, it can be seen that the transmission loss decreases from the initial $-0.1 \mathrm{~dB}$ down to $-0.06 \mathrm{~dB}$.

When the ground planes become too narrow $(g=250 \mu \mathrm{m})$ transmission loss does not further decrease due to the extra resistance introduced in the ground planes. Therefore, the results indicate that not only reflection but also transmission losses can be optimized with this impedance matching method. 


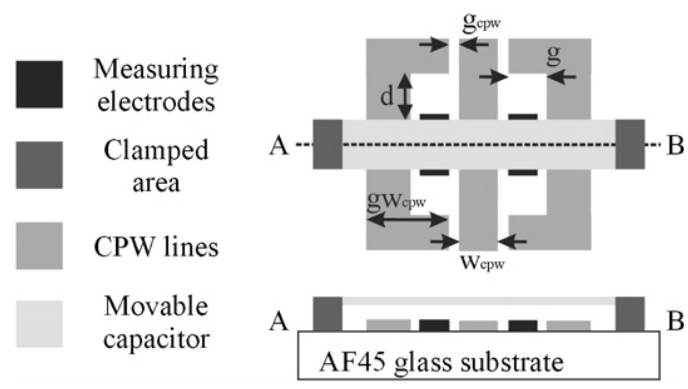

Figure 10. Schematic overview of a compensated floating RF power sensor design by moving ground planes apart (top view and cross section).

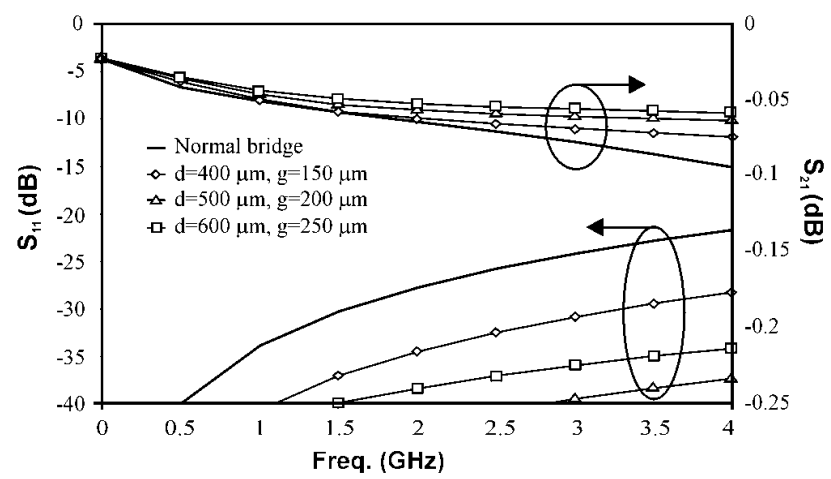

Figure 11. Simulation results for different designs of a compensated bridge sensor changing the width of the central line.

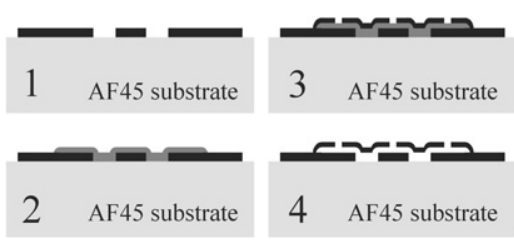

Figure 12. Outline of the fabrication process for the grounded-membrane sensor structure.

\section{Fabrication}

The fabrication of the sensor was done using aluminum surface micromachining on an AF45 substrate. This glass substrate was chosen due to its excellent RF properties $\left(\varepsilon_{\mathrm{r}}=6.2, \tan \delta=\right.$ $9 \times 10^{-4}$ at $1 \mathrm{MHz}$ ) [17].

\subsection{Grounded bridge}

For the fabrication of the 'grounded bridge', the process flow shown in figure 12 was used. First, a $1 \mu \mathrm{m}$ thick aluminum layer is sputtered and patterned in order to create the CPW lines and measuring electrodes.

After that, $1.7 \mu \mathrm{m}$ thick photoresist (Olin 907-17) is deposited and patterned in order to use it as a sacrificial layer. A baking step of $120{ }^{\circ} \mathrm{C}$ was then performed during $30 \mathrm{~min}$ in order for the polymer to solidify and allow the sputtering process on top of it. At the same time, the photoresist suffers a thermal reflow, resulting in a slightly thinner and smoother surface. A $1 \mu \mathrm{m}$ thick aluminum layer is then deposited and patterned by phosphoric acid using photoresist as a mask.

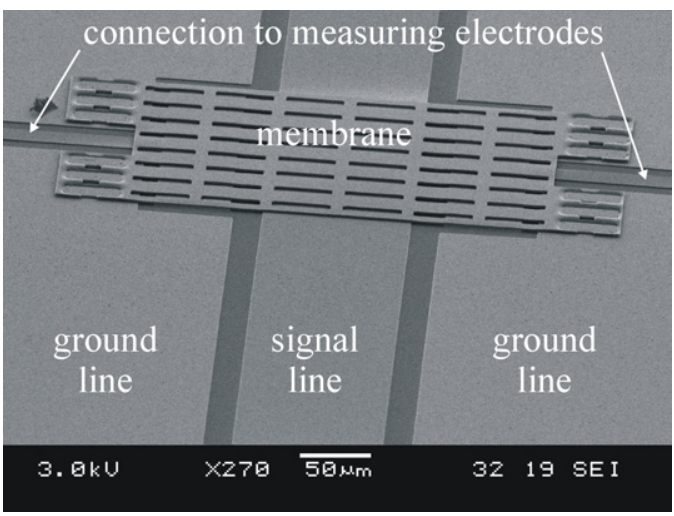

(a)

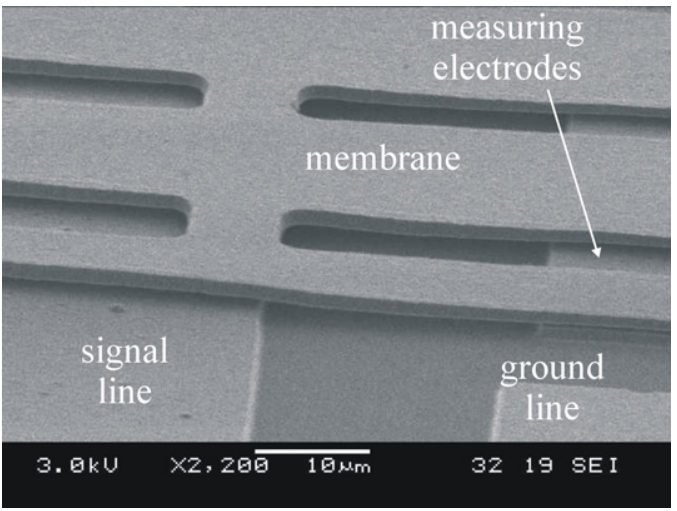

(b)

Figure 13. SEM photograph of the fabricated grounded-membrane sensor. The close-up $(b)$ clearly shows the curvature of the membrane due to the underlying CPW structure.

Finally, the membrane is released by oxygen plasma etching of the resist, since wet etching techniques would cause sticking problems of the membranes. SEM pictures of the fabrication results are shown in figure 13. As can be seen in figure 13(b), the use and the special treatment of the photoresist is not enough to completely planarize the sacrificial layer, and the shape caused by the difference in height of the CPW is still visible in the membrane. Nevertheless, no touching points are observed in the capacitor.

\subsection{Floating bridge}

In this case, longer membranes were designed with respect to the grounded bridges presented before. Therefore, the possibility of contact with the edges of the CPW increases due to the transferred pattern of the CPW. In order to avoid that, a new fabrication technique where the transmission lines are embedded into the glass substrate was developed. The process flow starts with the etching of $1 \mu \mathrm{m}$ deep recess by $1 \% \mathrm{HF}$ solution using a photoresist mask (see figure 14). Then, $800 \mathrm{~nm}$ of aluminum is deposited by sputtering. Removing the photoresist results in lift-off of the aluminum layer, giving an almost flat substrate with the coplanar waveguide and sense electrodes embedded in the surface. Finally, the movable plate is realized by aluminum surface micromachining using photoresist as the sacrificial layer. Microscope pictures with 


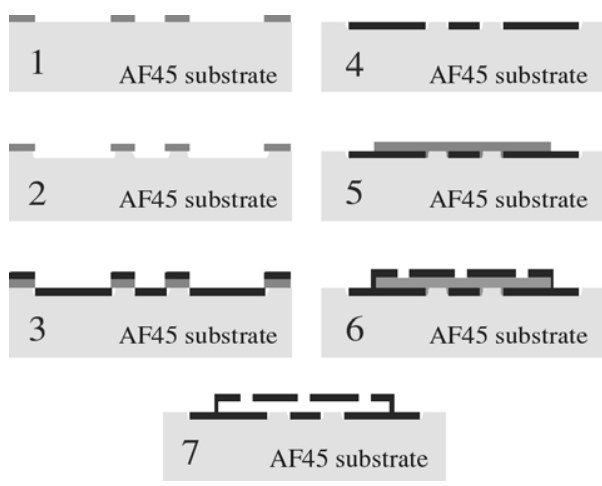

Figure 14. Outline of the fabrication process for the floating-membrane sensor structures. The CPW is embedded in the substrate to obtain a really flat membrane.

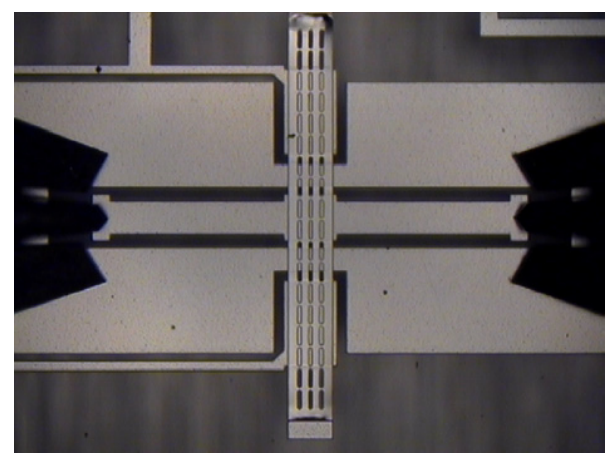

(a)

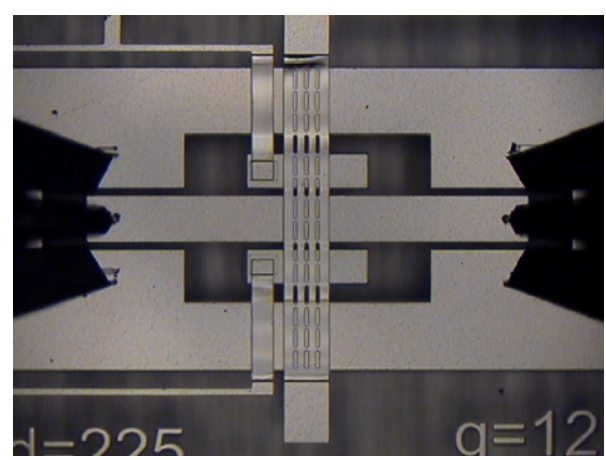

(b)

Figure 15. Microscope pictures showing floating power sensors being measured using RF probes: $(a)$ sensor with central line dimensions of the CPW changed (see figure 6); $(b)$ sensor with ground planes moved away (see figure 7).

the fabrication results for the two different kinds of impedance matching techniques are shown in figure 15. As can be observed from the SEM picture presented in figure 16(a), a very flat bridge is created on top of the CPW as a result of the embedding of the lines. Figure $16(b)$ shows a white light interferometer measurement along the length of the beam. Embedding the CPW in the substrate has reduced the steps in the bridge from $1 \mu \mathrm{m}$ (the thickness of the CPW lines) to $400 \mathrm{~nm}$. The peaks obtained were measured on top of the gap areas of the CPW and are caused by the difference in height between the cavity depth $(1 \mu \mathrm{m})$ and the refilling aluminum layer $(800 \mathrm{~nm})$.

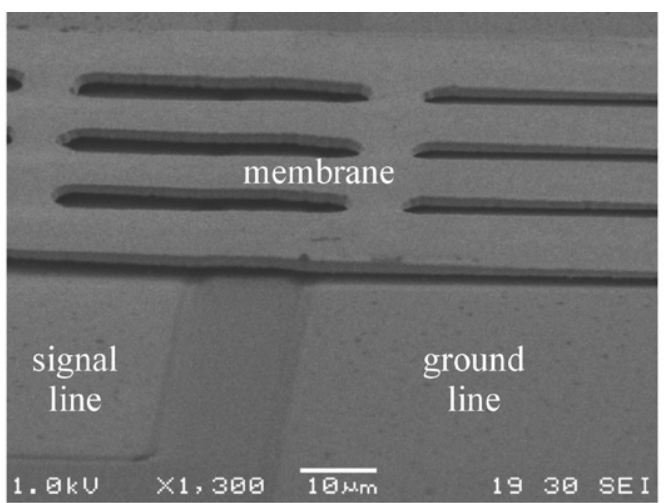

(a)

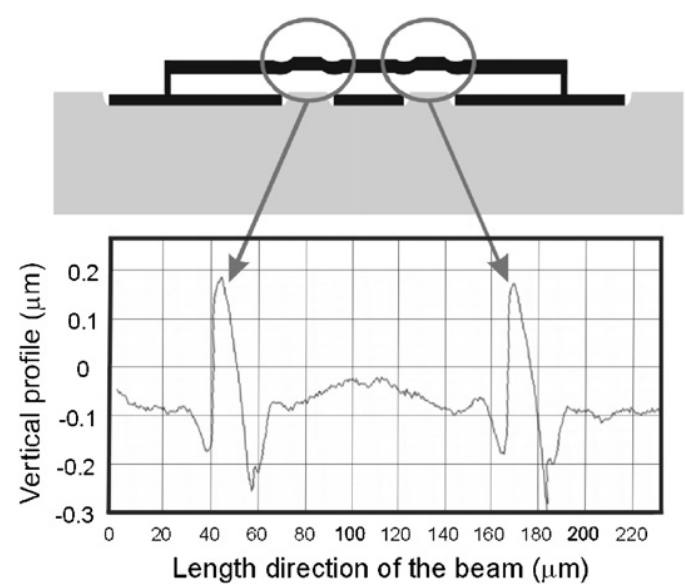

(b)

Figure 16. (a) SEM picture showing a floating bridge sensor with embedded CPW; $(b)$ white-light interferometer measurement showing the effectiveness of embedding the CPW in the substrate

\section{Measurement results}

\subsection{S-parameter measurements}

For the characterization of the RF response of the power sensor, measurements of $S$-parameters were performed up to $4 \mathrm{GHz}$ using a Vector Network Analyzer (RF source: HP83651, Controller: HP8510C and test-set: HP8517B) together with a Cascade Microtech 9000 probe. The socalled SOLT (short-open-load-through) calibration technique was used for the realization of the measurements.

6.1.1. Grounded-bridge power sensor. Figure 17(a) shows the insertion loss measurements from the different grounded bridge designs (symbols). Measurement results are compared with their theoretical estimations based on the extra capacitance expected from the geometrical dimensions of the different bridges and a constant gap of $1 \mu \mathrm{m}$ (lines). This leads to capacitance values of $3.2,1.6$ and $0.2 \mathrm{pF}$ for the 3600,1800 and 200 wide bridges, respectively. The measurement points of a simple CPW are shown as a reference. A good agreement between theory and experiments is found. Therefore, we can conclude that the reflection characteristics of the grounded bridge power sensor can be modeled by using an equivalent shunt capacitor introduced in the CPW, whose value can be estimated from the geometrical dimensions of the membrane. 


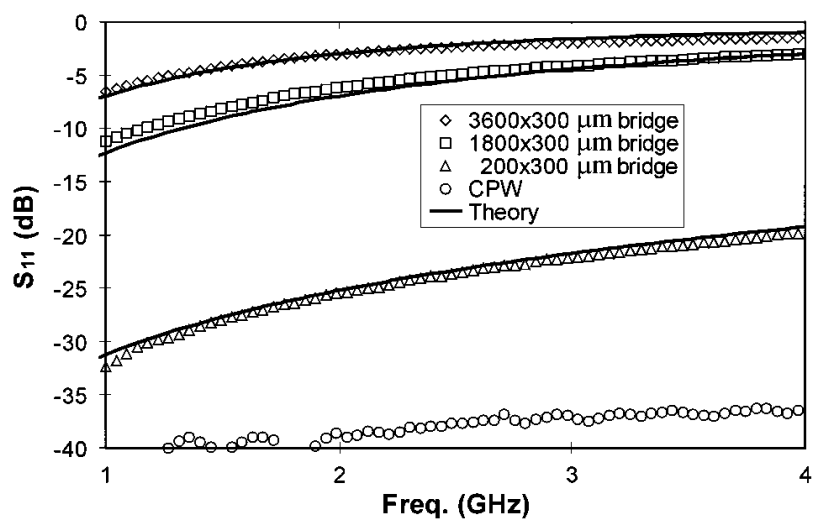

(a)

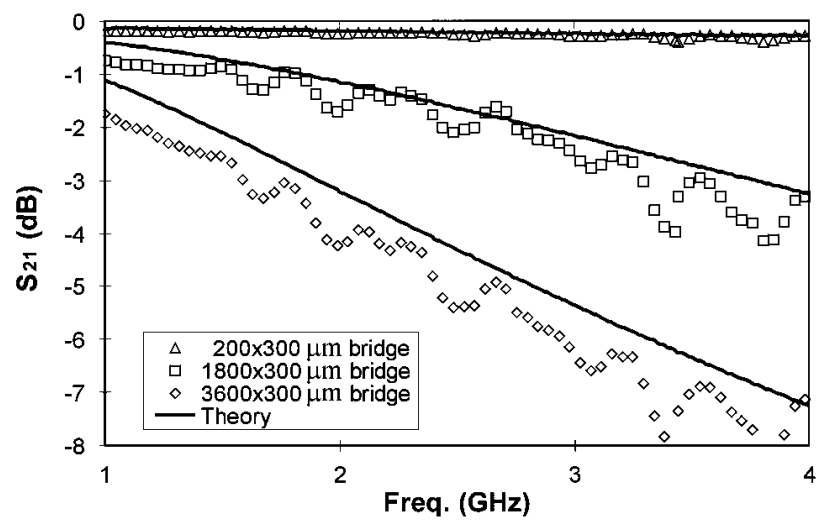

(b)

Figure 17. Measurements of the insertion loss expressed in $\mathrm{dB}$ on the grounded bridge sensors compared with the theoretical expectations.

In figure $17(b)$, the insertion losses for all three different grounded bridge sensors are shown. The lines represent the expected behavior calculated from the reflection losses of every specific sensor and the insertion losses measured on the CPW.

6.1.2. Floating-bridge power sensor. Figure 19 shows the measured return losses for the two different floating sensor designs (symbols). The results obtained are satisfactory modeled by the presence of a capacitance whose value is calculated from the geometrical dimensions of the different bridges and a gap distance of $0.6 \mu \mathrm{m}$, and a parasitic capacitance of $20 \mathrm{fF}$ (lines in figure 18(a)). The reduction of the gap distance with respect to the grounded bridge sensors is due to the reduction of the spring constant, which increases the initial deflection of the bridge by the residual tensile stress. This leads to capacitance values of 0.35 and $0.18 \mathrm{pF}$ for the 220 and $110 \mu \mathrm{m}$ wide bridges, respectively. The insertion losses measured on the two different floating bridge sensors are shown in figure 18(b) together with their theoretical expectation calculated from the reflection results presented in figure 18(a), and the insertion losses measured in the CPW. Slightly higher losses than expected are measured. The extra transmission losses measured are probably due to the extra resistive losses caused by the currents in the bridge [18]. Figure 18(b) shows lower transmission losses with respect

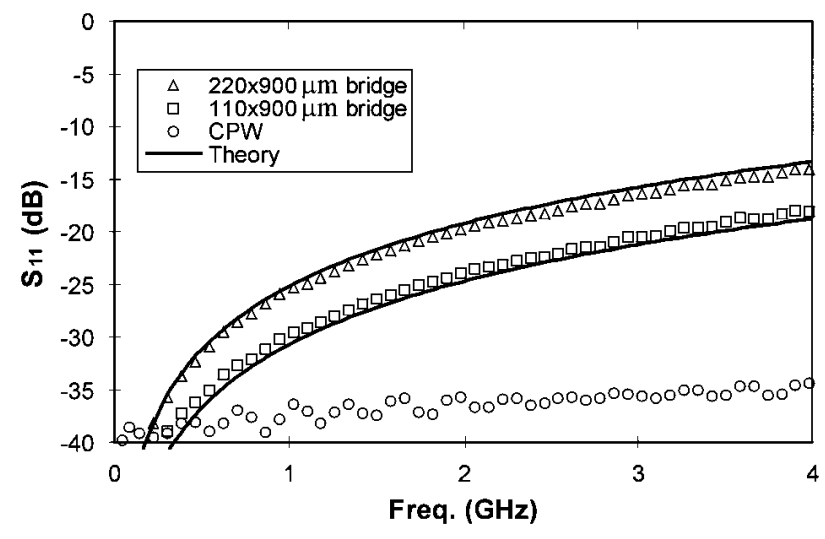

(a)

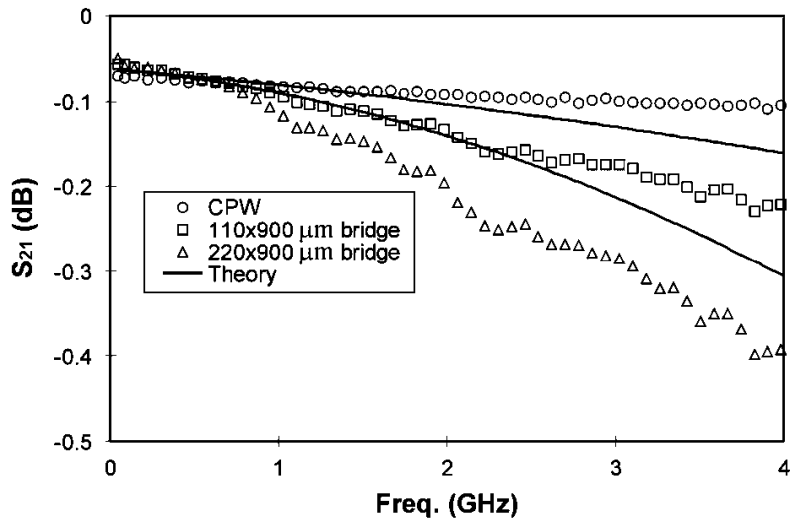

(b)

Figure 18. Measurement results on the floating bridge sensors compared with the theoretical expectations: $(a)$ return loss expressed in $\mathrm{dB} ;(b)$ insertion loss expressed in $\mathrm{dB}$.

to the grounded bridge designs as a consequence of the new dimensions of the sensor, resulting in $-0.2 \mathrm{~dB}$ insertion losses at $4 \mathrm{GHz}$ for the smallest floating bridge sensor.

In order to further improve this result, the compensation techniques presented in section 4.3 were implemented on the $110 \times 900 \mu \mathrm{m}$ floating bridge design. Figure 19(a) shows the results for the design in which the gap between the central line and the ground planes ' $g$ ' is modified by reducing the width of the signal line along a certain distance ' $d$ '. As can be observed, although lower reflections were obtained, higher insertion losses are measured. The smaller width of the signal line results in an increase of the resistance of the CPW, and therefore, in an increase of the insertion losses. We can conclude that reduction of the reflection losses can be obtained by this design, but no easy optimization of transmission losses seems to be possible. Figure 19(b) shows the measurement results of the sensor design where the gap distance ' $g$ ' is increased by partly removing the grounded lines of the CPW along a certain distance ' $d$ '. Reflection losses show an improvement of up to $10 \mathrm{~dB}$ at $4 \mathrm{GHz}$. Furthermore, an improvement in the transmission losses is observed. Only for the extreme situation with $d=250 \mu \mathrm{m}$ and $g=175 \mu \mathrm{m}$ we see that the transmission loss increases again. This is due to higher resistive losses introduced in the ground planes. We can conclude that both reflection and transmission losses can be optimized for this design by adjusting the dimensions $g$ and $d$. 


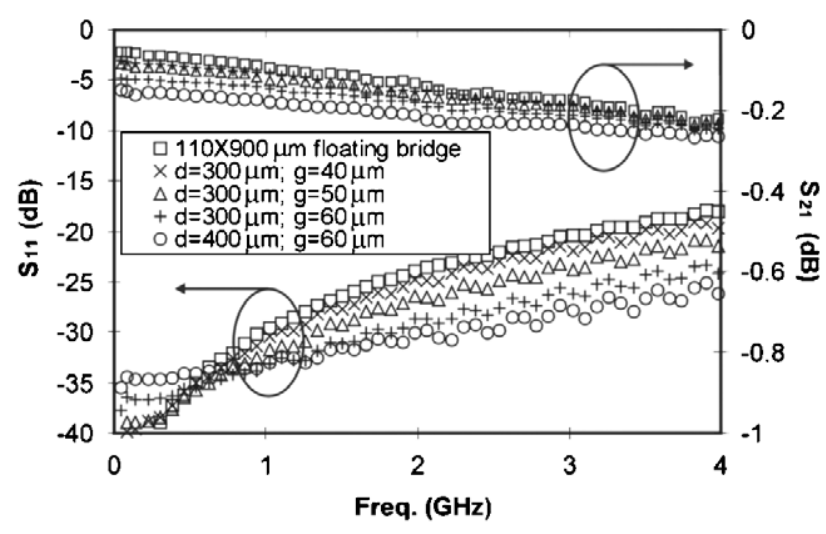

(a)

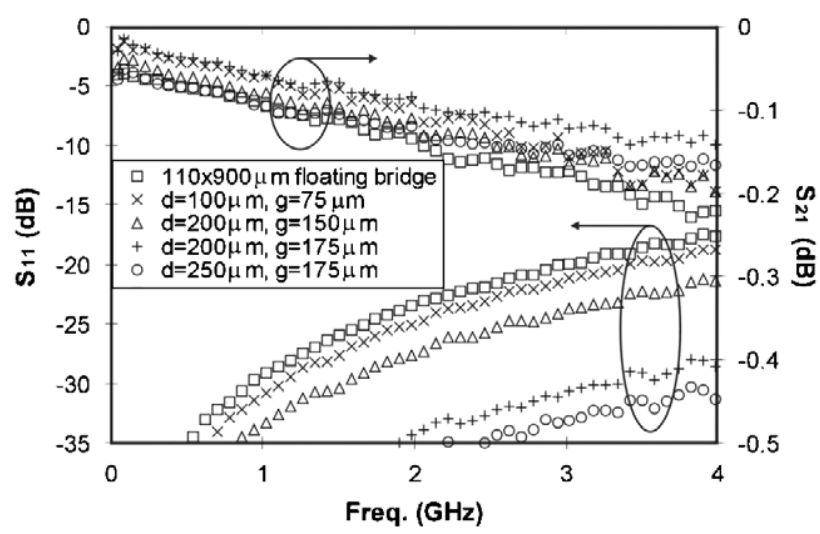

(b)

Figure 19. Experimental results of return and insertion losses from a $110 \times 900 \mu \mathrm{m}$ floating membrane with different CPW optimization techniques: $(a)$ reducing the width of the signal line; (b) modifying the width of the ground planes.

\section{2. $R F$ power sensing}

In order to realize capacitive RF power detection measurements with the bridge sensors, an RF source is used to deliver the signal that is to be detected. Then, a $50 \mathrm{~cm}$ long cable guides the signal to an RF probe (G-S-G PICO probes 40A, CS-5 from GGB Industries Inc.), which will introduce the signal into our bridge sensor. After passing through the power sensor, the signal travels to a second RF probe, which is again connected to a $50 \mathrm{~cm}$ long cable. Finally, the signal is absorbed by a commercially available RF power sensor (E4417A) to verify the presence of the RF signal at the bridge sensor. At the same time, the capacitance between the sensing electrodes and the bridge is detected by an impedance analyzer (4194A). In this way, we can measure capacitively the dependence between the movement of the bridge with respect to the power level and frequency of the RF signal traveling through the bridge sensor. The power level present on the bridge sensor was estimated by the average between the readout of the RF source and the power sensor placed at the end of the circuit. Then, the mismatch effect caused by the extra sensor capacitance (equation (5)) is used to obtain the final approximation to what the sensor really feels as RF signal.

6.2.1. Grounded-membrane power sensor. Due to the design of the grounded bridge, the sense capacitors are at one

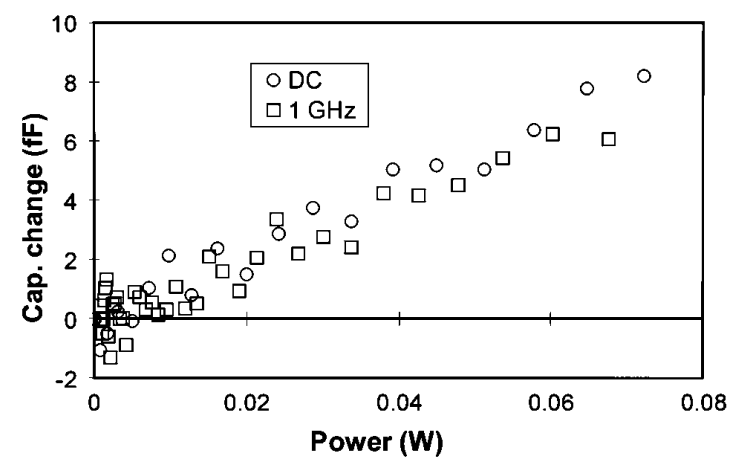

Figure 20. Measured capacitance change on the $3600 \times 300 \mu \mathrm{m}$ grounded bridge as a function of power for $1 \mathrm{GHz}$ RF signal and dc actuation.

side connected to ground. As a consequence, capacitance measurements suffer from parasitic capacitance and noise. As a result, only by using the biggest grounded bridge design ( $3.2 \mathrm{pF}$ bridge capacitance) the sensitivity was high enough to be detected by the impedance analyzer. Measurement results of the RF power detection for a $1 \mathrm{GHz}$ signal are shown in figure 20. As explained in section 2, the displacement of the bridge is linearly dependent on the power level of the RF signal. Because of the very small displacements, also a linear dependence between the power level and the change in sensing capacitance is observed. The results obtained with dc voltage actuation of the bridge are also included in the graph. In this case, the corresponding power level was estimated using the well-known relation $P=V^{2} / Z$, assuming an impedance of $50 \Omega$. A good agreement between the measurement results obtained from dc and RF actuation can be observed. In both cases a sensitivity in the order of $100 \mathrm{fF} \mathrm{W}^{-1}$ is obtained, which can also be calculated from the geometrical dimensions of the sensor assuming a tensile stress of $20 \mathrm{MPa}$ [12].

6.2.2. Floating-membrane power sensor. The movement detection of the floating bridge design was performed by measuring the capacitance change between the floating bridge and the sensing electrodes placed below. Since the capacitance to be measured is not electrically connected to ground, very low parasitic capacitance values and noise level were obtained, increasing the resolution of the measurement in comparison with the grounded bridge.

Figure 21 shows the capacitive change measured on the two different floating bridge designs when an RF signal of $1 \mathrm{GHz}$ is traveling through the sensor. The measurement results obtained for dc actuation are also included for reference. Again, a linear relation between the sensing capacitance and the power of the RF signal is found. Furthermore, the response of the sensor for dc and RF actuation agree with each other, confirming that the rms voltage squared of the signal was detected capacitively.

From figure 21 , a sensitivity of $90 \mathrm{fF} \mathrm{W}^{-1}$ for the $110 \times$ $900 \mu \mathrm{m}$ floating sensor and of $180 \mathrm{fF} \mathrm{W}^{-1}$ for the $220 \times$ $900 \mu \mathrm{m}$ floating sensor can be deduced. A tensile stress of $20 \mathrm{MPa}$ in the bridge causes the relatively small improvement of the sensitivity with respect to the grounded bridge. The resolution of the sensor is in the order of $0.5 \mathrm{~mW}$, which is still far from the theoretical noise limit as calculated in section 3.3. 


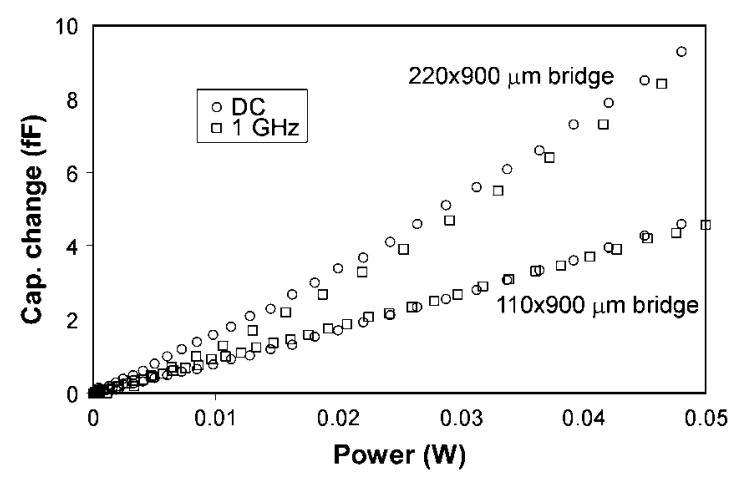

Figure 21. Measured capacitance change on $220 \times 900 \mu \mathrm{m} 110 \times$ $900 \mu \mathrm{m}$ floating bridge sensors as a function of power for a $1 \mathrm{GHz}$ signal and dc actuation.

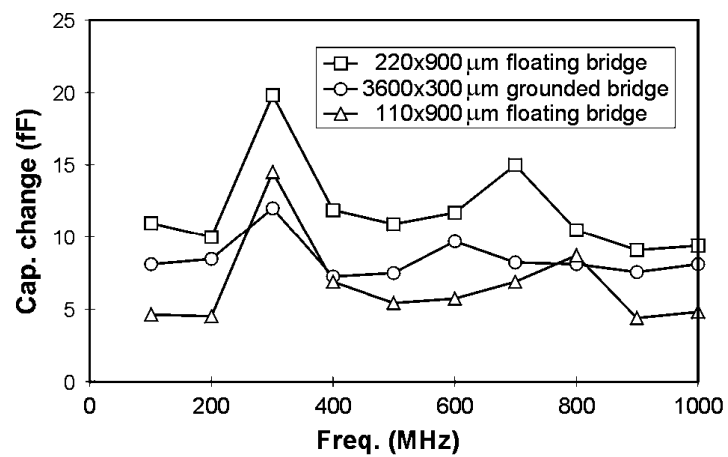

Figure 22. Measured capacitance change in the different bridge designs as a function of frequency for a fixed power level of $50 \mathrm{~mW}$ for the floating bridges and $80 \mathrm{~mW}$ for the grounded bridge.

6.2.3. Frequency dependence. The response of both grounded and floating sensors for different frequencies is illustrated in figure 22. As can be observed, a flat response as a function of frequency is found apart from two peaks. The first peak corresponds to $300 \mathrm{MHz}$ for all different devices. The second peak appears at $800 \mathrm{MHz}$ in the case of the $110 \times 900 \mu \mathrm{m}$ floating bridge and to $700 \mathrm{MHz}$ for the $220 \times$ $900 \mu \mathrm{m}$ floating bridge, whereas it was found at $600 \mathrm{MHz}$ for the grounded bridge. These peaks are most probably caused by the measurement set-up and the lack of calibration.

\section{Conclusions}

Wideband $100 \mathrm{kHz}-4 \mathrm{GHz}$ power sensors based on RF MEMS technology have been realized. The sensors are based on sensing the electrostatic force between the RF signal line and a suspended membrane. Furthermore, the sensors do not absorb the signal. Instead, 'through' sensors are realized, with almost no loss of signal. Fabrication was performed using aluminum surface micromachining. Embedding the CPW in the substrate improved the flatness of the membrane significantly. It was shown that a slight adjustment of the waveguide just before and after the sensor capacitance can be used to compensate for the sensor capacitance and thus improve reflection and transmission coefficients. An optimized bandwidth design resulted in return loss lower than $-28 \mathrm{~dB}$ and insertion loss lower than $0.15 \mathrm{~dB}$ at $4 \mathrm{GHz}$ and a sensitivity in the order of
$90 \mathrm{aF} \mathrm{mW}^{-1}$. The resolution was in the order of $0.5 \mathrm{~mW}$ and was limited by the capacitive detection of the bridge displacement.

\section{Acknowledgments}

This work is part of the IST-2000-28261 EMMA program of the European Commission. We wish to thank all EMMA members, VTT, MIKES, PTB, NMi, VTI and FLUKE, and $\mathrm{X}$ Rottenberg from IMEC.

\section{References}

[1] Tilmans H A C, De Raedt W and Beyne E 2003 MEMS for wireless communications: 'from RF-MEMS components to RF-MEMS-SiP' J. Micromech. Microeng. 13 S139-63A

[2] Seppä H, Kyynäräinen J and Oja A 2000 Microelectro-mechanical systems in electrical metrology IEEE Trans. Instrum. Meas. 50 440-4

[3] Elwenspoek M and Wiegerink R J 2001 Mechanical Microsensors (Berlin: Springer)

[4] Drieënhuizen B P and Wolffenbuttel R F 1995 Integrated electrostatic true RMS-to-DC converter IEEE Trans. Instrum. Meas. 44 370-3

[5] Suhonen M, Seppä H, Oja A S, Hweinilä M and Näkki I 1998 $\mathrm{AC}$ and $\mathrm{DC}$ voltage standards based on silicon micromechanics Proc. CPEM (Washington, DC, USA) pp 23-4

[6] Bartek M, Xiao Z, Van Mullem C and Wolffenbuttel R F 2000 Bulk-micromachined electrostatic RMS-to-DC converter: design and fabrication Proc. MME (Uppsala, Sweden)

[7] Kyynäräinen J, Oja A S and Seppä H 2000 A micromechanical RMS-to-DC converter Proc. CPEM (Sydney, Australia) pp 699-700

[8] Fernandez L J, Sesé J, Wiegerink R J, Flokstra J, Jansen H V and Elwenspoek M 2005 Radio frequency power sensor based on MEMS technology with ultra low losses Proc. MEMS'05 (Miami, USA)

[9] Fernandez L J, Visser E and Sese J et al 2004 Development of a capacitive MEMS RF power sensor without dissipative losses: towards a new philosophy of RF power sensing CPEM '04 (London, UK)

[10] Fernandez L J 2005 A capacitive RF power sensor based on MEMS technology $P h D$ Thesis University of Twente

[11] Vähä-Heikkilä T, Kyynäräinen J, Oja A, Varis J and Seppä H 2002 Capacitive MEMS power sensor Proc. of the 3rd Workshop on MEMS for Millimeterwave Communication (Crete, Greece)

[12] Pamidighantam S, Puers R, Baert K and Tilmans H A C 2002 Pull-in voltage analysis of electrostatically actuated beam structures with fixed-fixed and fixed-free end conditions $J$. Micromech. Microeng. 12 458-64

[13] Rottenberg X, Brebels S, De Raedt W, Nauwelaers B and Tilmans H A C 2004 RF-power: driver for electrostatic RF-MEMS devices J. Micromech. Microeng. 14 S43-8

[14] Nguyen C T-C 1994 Micromechanical signal processors PhD Thesis Berkeley University

[15] Agilent Technologies Impedance Measurements Handbook 2nd edn (Palo Alto, CA: Agilent Technologies) http://www.optics.arizona.edu/Palmer/OPTI380B/ PDFdocs/5950-3000.pdf

[16] www.sonnetusa.com

[17] http://www.schott.com/fpd/english/products/ fpd/af45.html

[18] Muldavin J B and Rebeiz G M 2000 High-isolation CPW MEMS shunt switches: 1. Modeling IEEE Trans. Microw. Theory Tech. 48 1045-52 